\title{
Fluorescence quenching of tryptophan and tryptophanyl dipeptides in solution ${ }^{*}$
}

\author{
Aaron P. Osysko ${ }^{1,2}$, Pedro L. Muíño ${ }^{1 \#}$ \\ ${ }^{1}$ Department of Chemistry, Saint Francis University, Loretto, USA; ${ }^{*}$ Corresponding Author: pmuino@ francis.edu \\ ${ }^{2}$ The Sherwin-Williams Company, Paints \& Coatings Division, Cleveland, USA.
}

Received 15 June 2011; revised 20 July, 2011; accepted 29 July 2011.

\begin{abstract}
We report measurements of fluorescence quantum yields of tryptophan, tryptophanylaspartate and tryptophanylarginine in several solvents as well as in aqueous solutions over a wide range of $\mathrm{pH}$. We aim to test a computational model developed by Callis and coworkers of fluorescence quantum yield, which postulates that quenching in tryptophan arises from energy loss due to an electron transfer from the aromatic system of tryptophan to one of the amides in the protein backbone. Since the electron transfer state is expected to be high in energy, normally this would not be a possible outcome, but because of its large dipole, such a state should be more accessible in polar solvents. In addition, conditions of low (high) pH, which result in a net positive (negative) charge for the terminal amine (carboxyl) should result in an increase (decrease) of electron transfer rates and low (high) quantum yields. The observed results confirm the predictions of the model.
\end{abstract}

Keywords: Tryptophan; Fluorescence; Quenching; Electron Transfer

\section{INTRODUCTION}

The amino acid tryptophan is unique in that its large size makes its spectroscopic properties significantly different from those of the other amino acids [1]. This property, coupled to its relative scarcity and to its sensitivity to the nature of the microenvironment, has made tryptophan a good probe of protein structure, which is the key parameter to understand protein functionality [2]. To this end, there are two spectroscopic properties of interest: fluorescence wavelength and fluorescence quantum yield. The effect of the environment on the

\footnotetext{
${ }^{*}$ This work was supported by NSF grant DUE-0525440.
}

fluorescence wavelength has been studied thoroughly using a combination of experimental and computational techniques [1-9]. As a result, the effect of the environment on the fluorescence maxima and spectral width is fairly well understood. This is the case even though environment effects can tune the fluorescence maxima of tryptophan in proteins to values within a $310-350 \mathrm{~nm}$ range, quantum yields from virtually 0 to 0.35 , and fluorescence lifetimes from 50 to 8000 ps [2,10-11]. But until recently, there has been no good model to relate quantum yield to the nature of the tryptophan microenvironment. In other words, there has been no clear way to make even qualitative predictions of fluorescence quantum yield for tryptophan in proteins.

The hypothesis that electron transfer from the indole chromophore to a backbone amide is responsible for low quantum yield scenarios has been present in the literature for a few decades [12-15]. Experimental support for this hypothesis is strongly provided by few, but very insightful experiments [14-16]. In the past decade, Callis and coworkers have computationally investigated the electronic landscape associated to this electron transfer mechanism [17-21]. According to this model, the presence of an electron transfer state in tryptophan (to a nearby amide), which is available in polar environments, may result in a quenching of the fluorescence (See Figure 1).

We aim to test this model by measuring the quantum yield of tryptophan, tryptophanylaspartate and tryptophanylarginine in solvents of different polarity. We also test it by measuring quantum yields in water at different $\mathrm{pHs}$. The expectation is that a low $\mathrm{pH}$ will create a positive charge in the terminal amine, thereby enhancing the probability of electron transfer and thus causing a lower quantum yield. A high $\mathrm{pH}$ environment will create a negative charge in the terminal carboxyl and thus decrease the probability of electron transfer. This situation should result in larger quantum yields.

Work on tryptophanyl dipeptides has been used very elegantly by Knutson and coworkers to investigate the 


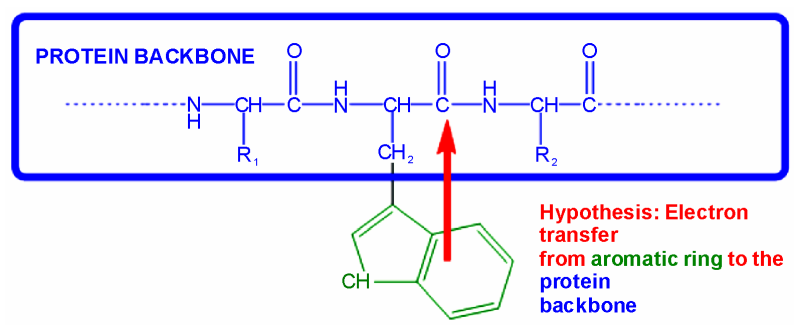

Figure 1. We test the hypothesis that electron transfer from the ring to an amide results in fluorescence quenching.

multiexponential decay of tryptophan in light of the rotamer model [22-23]. In these time resolved studies, the authors studied the multiexponential fluorescence decay in the picosecond regime and concluded that it is associated to ground state heterogeneity that may arise from different conformational rotamers of the peptide side chains.

Here we present experimental observations in aqueous solution ( $\mathrm{pH} \sim 2$ - 13), alcohols and acetonitrile.

\section{METHODS AND MATERIALS}

\subsection{Methods}

Absorption for each sample was collected using a Perkin Elmer UV/Vis Lambda 2S spectrophotometer. A Jobin-Yvon Horiba FluoroMax 3 was used to collect fluorescence data of tryptophan and the tryptophanyl dipeptides in solution.

The chromophores were dissolved in millipore water $(\mathrm{R} \geq 18.2 \mathrm{M} \Omega \mathrm{cm})$. The concentrations were kept below $0.1 \mathrm{mM}$, so that their absorbance maxima were under $\sim 0.1$. Samples of the solutions were placed into a Spectrosyl quartz cuvette (from Starna, with useable range $170-2700 \mathrm{~nm}$ ). Absorbances were measured at $280 \mathrm{~nm}$ in the UV/VIS spectrophotometer. After this, solutions of $0.01 \mathrm{M}, 0.1 \mathrm{M}$, or $2.5 \mathrm{M} \mathrm{NaOH}$; or $0.1 \mathrm{M}$ or $1.0 \mathrm{M}$ $\mathrm{HCl}$ were added dropwise to change the $\mathrm{pH}$ of the samples. Solutions of $\mathrm{pH} 2$ to 13 were prepared in this manner. Subsequently, the samples were returned to the volumetric flasks and bubbled with nitrogen for $10 \mathrm{~min}-$ utes to remove dissolved oxygen, which acts as a quencher. During the nitrogen purging, the flasks were placed in a $25^{\circ} \mathrm{C}$ water bath to maintain a constant temperature in all the solutions. After the ten minutes had elapsed, a sample of the solution was placed into $10 \mathrm{~mL}$ beaker in order to measure the final $\mathrm{pH}$. To this end, an electronic $\mathrm{pH}$ meter was calibrated using pre-made standards of $\mathrm{pH} 4.00,7.00$, and 10.00. After recording the $\mathrm{pH}$, each sample was placed in a cuvette and its absorption and fluorescence were sequentially collected: The absorption was measured at $280 \mathrm{~nm}$. Fluorescence was collected from 300 to $550 \mathrm{~nm}$ and resulting from a
$280 \mathrm{~nm}$ excitation (slit widths of $1 \mathrm{~mm}$ for both monochromators). The fluorescence signal used from here on refers to the integrated area of the fluorescence curve in the $300-550 \mathrm{~nm}$ range (baseline at zero), with the area of the fluorescence peak for the pure solvent, under the same conditions, subtracted. Prior to integration, the signal was corrected to account for the wavelength dependence of the detector's response. The same procedure (except for the $\mathrm{pH}$ adjustment) was followed for 3-methylindole (3MI) in water. Several researchers have reported slightly different values for the quantum yield of $3 \mathrm{MI}$ in water. We have chosen 0.34 as the value for the standard for the measurement of the quantum yield, based on [24] $\left(0.34\right.$ at $\left.25^{\circ} \mathrm{C}\right)$ and [25] $\left(0.34\right.$ at $\left.20^{\circ} \mathrm{C}\right)$. Because only a geometric fraction of the fluorescence was collected, this approach was used in lieu of a full solid angle measurement of the fluoresence. The quantum yield $\left(\Phi_{f}\right)$ of each sample was then obtained from the relative yield of tryptophan or the dipeptides $\left(I_{T R P}\right)$ to the yield of $3 \mathrm{MI}\left(I_{3 M I}\right)$. Each of these is, in turn, obtained from the ratio between fluorescence and absorption:

$$
\begin{aligned}
\Phi_{f} & =0.34\left(I_{T R P} / I_{3 M I}\right) \\
& =0.34\left(F_{T R P} / A_{T R P}\right) *\left(A_{3 M I} / F_{3 M I}\right)
\end{aligned}
$$

A minimum of three independent set of measurements were performed for each solution and the quantum yields averaged. In the case of the other solvents, the millipore water was simply replaced with the solvent and the same procedure was followed.

\subsection{List of Materials}

Tryptophan: 12729HS, 73-22-23, Aldrich Chemical Company, 99\%.

3-Methylindole: 83-34-1, Lot No. 08816DR, M5 145-8, Aldrich Chemical Company, 99\%.

Tryptophanylaspartate: Lot No. 117953, Bachem California Inc., $98 \%$.

Tryptophanylarginine: Lot No. 508139, Bachem California Inc., 98\%.

Millipore Water: $\geq 18.2 \mathrm{M} \Omega$.

Methanol: CAS No. 67-56-1, Science Kit \& Boreal Laboratories, $100 \%$.

1-Butanol: Lot No. 740530, Fisher Scientific Company, $99.9+\%$.

1-Octanol: CAS No. [111-87-5], Lot No. KB 01345KB, Cat. No. 11,261-5 EEC No. 203-917-6, Sigma-Aldrich Chemical Company, 99\%.

Hexanol: CAS No. 11-27-3, H13303-1L, Batch No. 14723K1, EEC No. 203-852-3 Sigma-Aldrich Chemical Company, $98 \%$.

Acetonitrile: CAS No. 75-050-8, Stock No. 42879, Lot No. G14Q15, Alfa Aesar, 99.9+\%. 


\section{RESULTS}

The measured quantum yields of the aqueous solutions as a function of $\mathrm{pH}$ are reported in Figure 2. All three systems display a similar pattern with low quantum yields in the cationic state (low $\mathrm{pH}$ ). The quantum yield increases with $\mathrm{pH}$ as the system transitions via the zwitterionic to the anionic state. The maximum value of the quantum yield actually depends on the nature of the chromophore, with tryptophan and tryptophanylaspartate showing strong fluorescence and tryptophanylarginine having maximum values near those of 3MI. At extremely high $\mathrm{pH}$ values, all three systems see a drop off in quantum yield. See the Discussion section for the interpretation of these results.

To aid in the comparison of the quantum yield values, Figure 3 shows a fit of the Figure 2 data. This was obtained using a polynomial fit in Origin 7.0. Nevertheless, we do not attribute any specific significance to the actual polynomial parameters. Rather, the goal is to show a basis of comparison for the three systems studied regarding the relative quantum yields and the $\mathrm{pH}$ values at which quantum yields start to qualitatively change.

Table 1 summarizes the quantum yields for the three chromophores in the alcohols and acetonitrile.

\section{DISCUSSION}

The data collected clearly shows that cationic conditions (low $\mathrm{pH}$ ) result in a very low quantum yield. This is consistent with the predictions of the model. Indeed, if the fluorescence lifetime $\left(\tau_{f l}\right)$ is modeled from the rate constants associated to radiative decay $\left(k_{r}\right)$, nonradiative decay $\left(k_{n r}\right)$ and electron transfer $\left(k_{e t}\right)$, we can write, as in $[18,19]$, and [21],

$$
\tau_{f l}=\left(k_{r}+k_{n r}+k_{e t}\right)^{-1}
$$

Consequently, the quantum yield is the percentage of energy loss that takes place via the radiative pathway, and can be expressed as:

$$
\Phi_{f}=k_{r} /\left(k_{r}+k_{n r}+k_{e t}\right)
$$

Therefore, a positively charged terminal amine in tryptophan or the dipeptides serves to enhance the probability of an electron transfer event from the indole ring to the amide group (in the dipeptides, not in tryptophan), thus increasing $k_{e t}$. As the $\mathrm{pH}$ increases, more terminal carboxyls acquire negative charge and the electron transfer becomes more problematic. Thus, the radiative pathway becomes more likely as the means for energy loss and the quantum yield increases. In this sense, the transition from low to high $\mathrm{pH}$ values shows the typical profile of a titration curve.

We chose to use dipeptides with the second amino

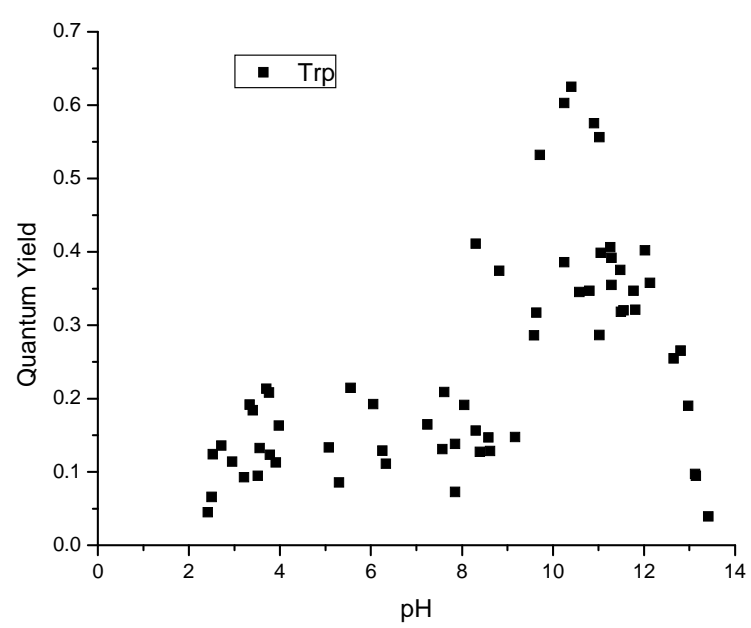

(a)

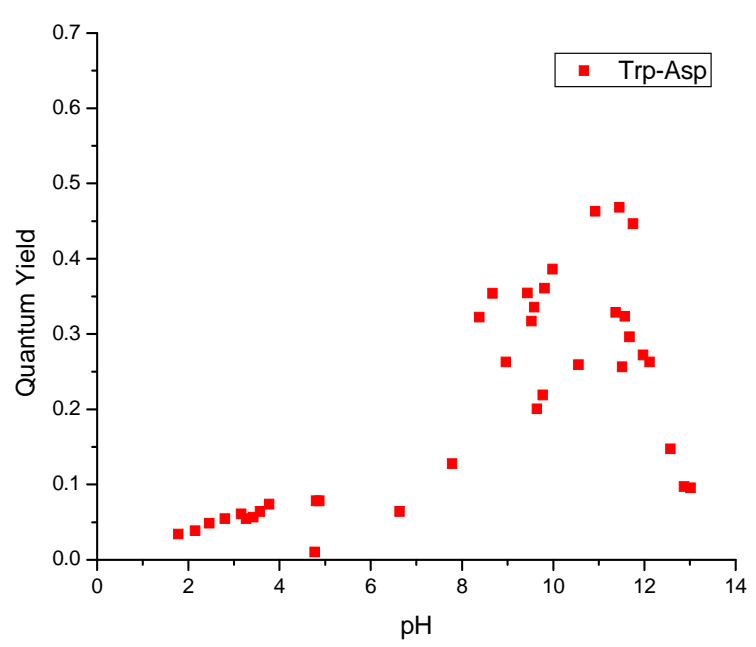

(b)

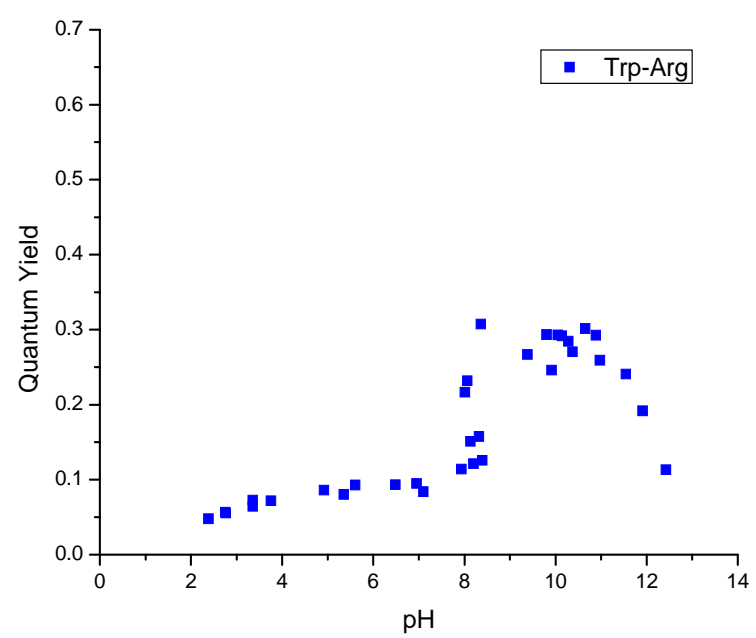

(c)

Figure 2. Quantum yields as a function of $\mathrm{pH}$ for (a) tryptophan (top pane); (b) tryptophanylaspartate (middle pane) and (c) tryptophanylarginine (bottom pane). 


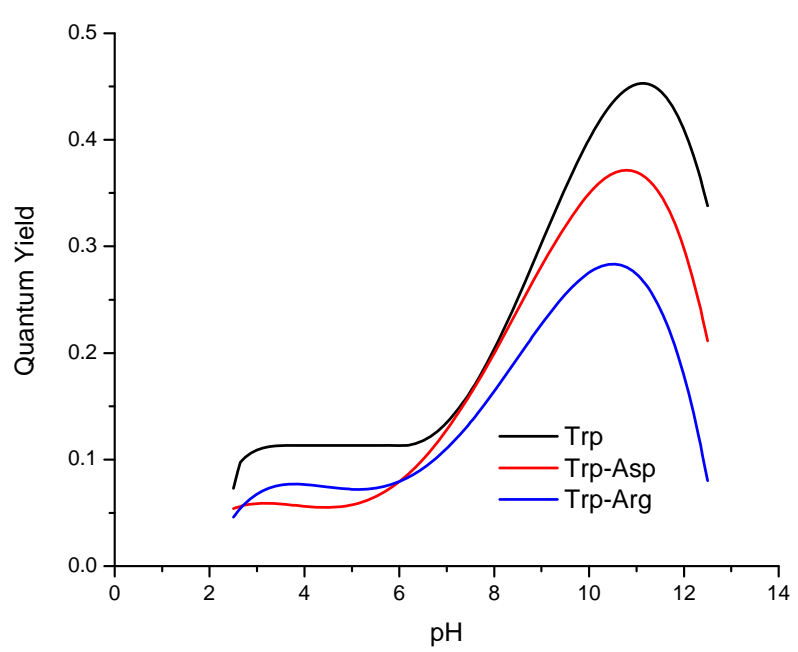

Figure 3. Comparison of the fitted quantum yields for tryptophan, tryptophanylaspartate, and tryptophanylarginine. Fits obtained using OriginPro 7.0.

Table 1. Quantum yield of tryptophan and the dipeptides in the non-aqueous solvents. The dipeptides did not dissolve in acetonitrile.

\begin{tabular}{cccc}
\hline & Tryptophan & Trp-Asp & Trp-Arg \\
\hline Methanol & $0.14 \pm 0.02$ & $0.17 \pm 0.07$ & $0.12 \pm 0.02$ \\
1-Butanol & $0.06 \pm 0.10$ & $0.37 \pm 0.17$ & $0.33 \pm 0.15$ \\
1-Hexanol & $0.14 \pm 0.03$ & $0.18 \pm 0.01$ & $0.33 \pm 0.23$ \\
1-Octanol & $0.09 \pm 0.03$ & $0.06 \pm 0.04$ & $0.32 \pm 0.04$ \\
Acetonitrile & $0.36 \pm 0.05$ & - & - \\
\hline
\end{tabular}

acid carrying a charged side chain in order to evaluate the effect of this factor on the electron transfer rate. As expected, the presence of the second amino group in tryptophanylarginine results in a lower overall quantum yield. Even at high $\mathrm{pH}$ values, the group is significantly charged (the pKa of the side chain is 12.48 for arginine [26]) and the quantum yield is significantly lower than for the other two systems. This is predicted by the model because the positive charge stabilizes the electron transfer from the indole ring to the amide. This means that the electron transfer state, which is, in general, significantly above the fluorescing state [17] can become near resonant with the latter under these circumstances.

Blancafort et al. have addresed, via very thorough computational work, the quenching in tryptophan at low $\mathrm{pH}$ [27]. They document a reaction pathway where the positively charged amine group forms an intramolecular hydrogen bond to $\mathrm{C} 4$, with a subsequent proton transfer to the indole ring. Radiationless decay would take place at a conical intersection resulting from a pathway that bifurcates towards photodecarboxylation and photo- tautomerization routes. It should be noted, however, that the photodecarboxylation route is not available for the dipeptides, which lack an unbound carboxyl group in the tryptophan moiety.

Both tryptophan and tryptophanylaspartate have higher quantum yields than tryptophanylarginine. In fact, tryptophan shows larger values in the fitted curve than tryptophanylaspartate, which is a slightly puzzling result in view of our hypothesis. This would predict that the negative charge would hinder electron transfer and increase the fluorescence quantum yield. On the other hand, indole, which does not have an amide moiety that can accept the electron, has a quantum yield of 0.34 and the higher values observed here must be related to the effect of the additional microenvironment on the energy levels of indole ring. In fact, the theoretical model offers reasonably good quantitative predictions by ascribing all the variability in quantum yield to the change in $k_{e t}$ while assuming constant values [18] for $k_{r}$ and $k_{n r}$ in (3). Presumably, the microenvironment does affect the value of these two constants to some extent in the systems studied here.

The quantum yield values begin to decrease as the highest $\mathrm{pH}$ levels are reached. We attribute this to the deprotonation of the NH group in the indole. Sobolewski and Domcke have done detailed ab initio calculations that show that the proton detachment leads to an efficient mechanism for non radiative decay [28].

Work with the other solvents aimed to provide further testing of the hypothesis. Due to the electronic nature of the charge transfer state, it would be expected that solvents capable of hydrogen bonding would lower the state's energy, resulting in a lower quantum yield. Indeed, previous work [20] shows that this trend holds for $\mathrm{N}$-acetyltryptophanamide (NATA). NATA is a proxy for tryptophan in a protein, having two amide units capable of accepting the electron transferred. In that work, quantum yields went from 0.13 (in water) to 0.36 (in dioxane) with alcohol solvents resulting in values clustering around 0.2. We expected equivalent behavior in the current work and the results were somewhat similar, with the alcohols clustering around values of 0.15 . The exceptions are trp-arg in butanol or hexanol and trp-asp in butanol, which show higher values, albeit with very high error bars. Tryptophan does show a high quantum yield in acetonitrile, but the dipeptides did not dissolve in this solvent.

\section{CONCLUSIONS}

We aimed to test the hypothesis presented by Callis and coworkers that electron transfer from the indole ring to one of the amides in the protein backbone is the main mechanism for fluorescence loss in proteins (low quan- 
tum yield). We observed that high $\mathrm{pH}$ results in a high quantum yield because the negatively charged carboxyl results in a very low efficiency for the electron transfer events. Low $\mathrm{pH}$ does result in a low quantum yield. In the dipeptides, a positively charged amine group is expected to lower quantum yield by increasing the rate of electron transfer to the amide. This mechanism is not available for tryptophan (no amide). Whether or not the same mechanism is responsible for the low quantum yield at low $\mathrm{pH}$ in all three systems (tryptophan and the two dipeptides), the experimental observations are compatible with the electron transfer hypothesis. The model does not address the observed decrease in quantum yield at extremely high $\mathrm{pH}$ values, which we interpret as related to the deprotonation of the $\mathrm{NH}$ group in the indole. It is also observed that the effect of the charge in the side chains (aspartate is negatively charged, arginine is positively charged) affects the overall quantum yield. For instance, the maximum quantum yield in tryptophanylarginine is lower than in tryptophan, possibly because the positive charge prevents the electron to be transferred in the direction of the amide. The correlation between (low) quantum yields and solvent polarity has also been hinted at by these data. However, more experiments are necessary to explain some inconsistent data from the alcohols. Also, these experiments should include solvents of lower polarity that can dissolve these chemicals. These findings are consistent with a model that can be used to make predictions of local protein structure, a key component in the functionality of the protein.

\section{ACKNOWLEDGEMENTS}

We thank Dr. Balazs Hargittai for his help interpreting the $\mathrm{pH}$ effects.

\section{REFERENCES}

[1] Demchenko, A.P. (1986) Ultrasiolet spectroscopy of proteins. Springer-Verlag, New York.

[2] Eftink, M.R. (1991) Fluorescence techniques for studying protein structure. Methods of Biochemical Analysis, 35, 127-205. doi:10.1002/9780470110560.ch3

[3] Weber, G. (1960) Fluorescence-polarization spectrum and electronic energy transfer in tyrosine, tryptophan and related compounds. Biochemical Journal, 75, 335-345.

[4] Konev, S.V. (1967) Fluorescence and phosphorescence of proteins and nucleic acids. Plenum, New York.

[5] Beechem, J.M. and Brand, L. (1985) Time-resolved fluorescence of proteins. Annual Review of Biochemistry, 54, 43-71. doi:10.1146/annurev.bi.54.070185.000355

[6] Muíño, P.L. and Callis, P.R. (1994) Hybrid simulations of salvation effects on electronic spectra: Indoles in water. Journal of Chemical Physics, 100, 4093-4109.

[7] Callis, P.R. (1997) ${ }^{1} \mathrm{~L}_{\mathrm{a}}$ and ${ }^{1} \mathrm{~L}_{\mathrm{b}}$ transitions of tryptophan: Applications of theory and experimental observations to fluorescence of proteins. Methods in Enzymology, 278, 113-150. doi:10.1016/S0076-6879(97)78009-1

[8] Chen Y. and Barkley, M.D. (1998) Toward understanding tryptophan fluorescence in proteins. Biochemistry, 37, 9976-9982. doi:10.1021/bi980274n

[9] Vivian, J.T. and Callis, P.R. (2001) Mechanisms of tryptophan fluorescence shifts in proteins. Biophysical Journal, 80, 2093-2109. doi:10.1016/S0006-3495(01)76183-8

[10] Toptygin, D., Savtchenko, R.S., Meadow, N.D. and Brand, L. (2001) Homogeneous spectrally- and time-resolved fluorescence emission from single-tryptophan mutants of IIA $^{\text {Glc }}$. Journal of Physical Chemistry B, 105, 2043-2055. doi:10.1021/jp003405e

[11] Xu, J., Chen, J., Toptygin, D., Tcherkasskaya, O., Callis, P.R., King, J., Brand, L. and Knutson, J.R. (2009) Femtosecond fluorescence spectra of tryptophan in human $\gamma$-crystallin mutants: Site-dependent ultrafast quenching. Journal of the American Chemical Society, 131, 1675116757. doi:10.1021/ja904857t

[12] Cowgill, R.W. (1970) Fluorescence and protein structure. XVII. On the mechanism of peptide quenching. Biochimica et Biophysica Acta, 200, 18-25.

[13] Feitelson, J. (1970) Environmental effects on the fluorescence of tryptophan and other indole derivatives. Israel Journal of Chemistry, 8, 241-252.

[14] Petrich, J.W., Chang, M.C., McDonald, D.D. and Fleming, G.R. (1983) On the origin of nonexponential fluorescence decay in tryptophan and its derivatives. Journal of the American Chemical Society, 105, 3824-3832. doi:10.1021/ja00350a014

[15] Chen, Y., Liu, B., Yu, H.T., Barkley, M.D. (1996) The peptide bond quenches indole fluorescence. Journal of the American Chemical Society, 118, 9271-9278. doi:10.1021/ja961307u

[16] Sillen, A., Hennecke, J., Roethlisberger, D., Glockshuber, R. and Engelborghs, Y. (1999) Fluorescence quenching in the DsbA protein from E. coli: Complete picture of the excited-state energy pathway and evidence for the reshuffling dynamics of the microstates of tryptophan. Proteins: Structure, Function, and Genetics. 37, 253-263. doi:10.1002/(SICI)1097-0134(19991101)37:2<253::AID -PROT10>3.0.CO;2-J

[17] Vivian, J.T. and Callis, P.R. (2002) Understanding the variable fluorescence quantum yield of tryptophan in proteins using QM-MM simulations. Quenching by charge transfer to the peptide backbone. Chemical Physics Letters, 369, 409-414.

[18] Callis, P.R., Liu, T. (2004) Quantitative prediction of fluorescence quantum yields for tryptophan in proteins. Journal of Physical Chemistry B, 108, 4248-4259. doi:10.1021/jp0310551

[19] Callis, P.R., Petrenko, A., Muíño, P.L., Tusell, J.R. (2007) $A b$ Initio prediction of tryptophan fluorescence quenching by protein electric field-enabled electron transfer. Journal of Physical Chemistry B, 111, 10335-10339. doi:10.1021/jp0744883

[20] Muíño, P.L. and Callis, P.R. (2009) Solvent effects on the fluorescence quenching of tryptophan by amides via electron transfer. Experimental and computational studies. Journal of Physical Chemistry B, 113, 2572-2577. doi:10.1021/jp711513b

[21] Pan, C.P., Muíño, P.L., Barkley, M.D. and Callis, P.R. 
(2011) Correlation of tryptophan fluorescence spectral shifts and lifetimes arising directly from heterogeneous environment. Journal of Physical Chemistry B, 115, 3245-3253. doi:10.1021/jp111925w

[22] Chen, R., Knutson, J.R., Ziffer, H. and Porter, D. (1991) Fluorescence of tryptophan dipeptides: Correlations with the rotamer model. Biochemistry, 30, 5184-5195. doi:10.1021/bi00235a011

[23] Xu, J. and Knutson, J.R. (2009) Quasi-static self-quenching of Trp-X and X-Trp dipeptides in water: Ultrafast fluorescence decay. Journal of Physical Chemistry B, 113, 12084-12089. doi:10.1021/jp903078x

[24] Yu, H.T., Colucci, W.J., McLaughlin, M.L. and Barkley, M.D. (1992) Fluorescence quenching of indoles by excited state proton transfer. Journal of the American Chemical Society, 114, 8449-8454. doi:10.1021/ja00048a015

[25] Eftink, M.R., Jia, Y., Hu, D. and Ghiron, J.A. (1995)
Fluorescence studies with tryptophan analogs: Excited state interactions involving the side chain amino group. Journal of Physical Chemistry, 99, 5713-5723. doi:10.1021/j100015a064

[26] Dawson, R.M.C., Elliot, D.C., Elliot, W.H. and Jones, K. M. (1986) Data for biochemical research. Clarendon Press, Oxford.

[27] Blancafort, L., González, D., Olivucci, M., Robb, M.A. (2002) Quenching of tryptophan ${ }^{1}\left(\pi, \pi^{*}\right)$ fluorescence induced by intramolecular hydrogen abstraction via an aborted decarboxylation mechanism. Journal of the American Chemical Society, 124, 6398-6406. doi:10.1021/ja016915a

[28] Sobolewski, A.L. and Domcke, W. (1999) Ab initio investigations on the photophysics of indole. Chemical Physics Letters, 315, 293-298. doi:10.1016/S0009-2614(99)01249-X 